\title{
Review Talk on Field Theory
}

\author{
Victor O. Rivelles \\ Instituto de Física, Universidade de São Paulo \\ Caixa Postal 66318, 05315-970, São Paulo, SP, Brazil
}

Received on 11 August, 2006

\begin{abstract}
I will compare the field theory works presented during the 2005 Encontro Nacional de Física de Partículas e Campos with what is done in the rest of the world in this area.
\end{abstract}

Keywords: Quantum Field Theory

In 2003 the review talk was present by Dionisio Bazeia Filho from UFPb. He did a necessary and very interesting comparison on the number of works presented in each subarea of field theory, and the corresponding number of works posted at the arXives. Large distortions were then detected. Most of the works presented at that meeting, $15 \%$, were in field theory applied to condensed matter while in the arXives this proportion is just $1 \%$. The most active area in the arXives, string theory, with $25 \%$ of the submissions, corresponded to just $8 \%$ of the works presented at the meeting.

I do not intend to update the table presented by Dionisio. We would not find significative changes in two years. However, it is interesting to compare what is done in the rest of the world with what is being done in Brazil.

Today, most of the researchers in field theory work in string theory abroad. In Brazil, however, there is a large community which is dedicated to the study of field theory without worrying with its string theory aspects. This is worrisome since it lacks modernity to the research being done and keeps the researchers alienated with regard to the advances produced by string theory.

The perturbative aspects of field theory are fully understood. Either in four or two dimensions. Unfortunately, there is a considerable number of researchers in this area in Brazil. The only research line which still needs some understanding is the calculation of scattering amplitudes, mainly in non-abelian gauge theories [1]. Techniques which allow the calculation of amplitudes for many particles or involving several loops are still in development. String theory techniques are extremely useful.

The real big problem in gauge theory is the understanding of its non-perturbative sector. The spectrum of non-abelian gauge theories, confinement of QCD, Gribov copies, 1/N expansion and calculations in the lattice are the main topics abroad. Fortunately, many Brazilian groups are dedicated to these problems.

Another well spread area in Brazil is the study of noncommutative field theories. There are several groups working in several aspects in many regions of Brazil. Also important is field theory in curved spaces. Some Brazilian groups have interest in this topic. Field theory at finite temperature is another line in which we have several groups, but abroad there is not much going on. Applications to condensed matter is another line in the same situation. However, the Casimir effect and the use of boundary conditions in field theory represent lines with a large amount of Brazilians involved and which also have a similar development abroad.

A significative number of the community is dedicated to the study of integrability in field theory, mainly in two dimensions. It must be noticed that today there are some signs that the maximally supersymmetric gauge theory is integrable. There is a strong connection with integrability in string theory in some backgrounds [2]. Unfortunately nobody in Brazil is dedicated to this line.

A new area opened by string theory and by loop quantum gravity is the study of violation of Lorentz symmetry. It is not really a violation of Lorentz symmetry but rather the study of field theories in which some vector or tensor field acquires an vacuum expection value [3]. It is an expanding area abroad and it is attracting some people in Brazil.

No doubt, string theory represents the next step to be followed after the success of field theory [4]. However, the number of Brazilian researchers in this area is extremely reduced. It is a very dynamical and competitive field and maybe this explains the retraction of our community. Amplitude calculations is one of the few areas being developed in Brazil. String field theory, matrix models, and topological string theory simply do not exist in Brazil but have great activity abroad. String phenomenology, and mainly the study of landscape, are other research lines with great activity abroad but only now some Brazilian researchers have shown some interest. The several cosmological models bases in string theory, like braneworlds, Randall-Sundrum models, extra dimensions and effective actions have some Brazilian groups involved and it seems they are consolidating their work.

However, the great activity today is in the study of the AdS/CFT correspondence, or more generically, in the correspondence of gravitation and gauge theory. Getting the spectrum of gauge theories using the correspondence involves some Brazilians. As mentioned before, the line which is growing nowadays is the search for integrability on both sides of the correspondence. However, this line is not yet being developed here.

I hope to have drawn a profile of the works presented in this meeting, comparing them with the most important lines that are being developed abroad. We have several groups involved in these more dynamical lines but we still have a significative number of people working in topics which are not very important. I hope that in a not so short time this picture will change so that we can contribute for the solution of the big problems of our area.

I will just provide some basic references. 
[1] Z. Bern, "Recent progress in perturbative quantum field theory. ((U)) ((W))," Nucl. Phys. Proc. Suppl. 117, 260 (2003) [arXiv:hep-ph/0212406].

[2] J. Plefka, "Spinning strings and integrable spin chains in the AdS/CFT correspondence," arXiv:hep-th/0507136.

[3] D. Colladay and V. A. Kostelecky, "Lorentz-violating exten- sion of the standard model," Phys. Rev. D 58, 116002 (1998) [arXiv:hep-ph/9809521].

[4] J. de Boer, "String theory: An update," Nucl. Phys. Proc. Suppl. 117, 353 (2003) [arXiv:hep-th/0210224]. 